\title{
IMPACTO DA COVID-19 SOB O TRABALHO DA ENFERMAGEM BRASILEIRA: ASPECTOS EPIDEMIOLÓGICOS
}

Vagner Ferreira do Nascimento ${ }^{1}$ Mariano Martinez Espinosa ${ }^{2}$ Manoel Carlos Neri da Silva ${ }^{3}$ Neyson Pinheiro Freire ${ }^{3}$ Ana Cláudia Pereira Terças Trettel ${ }^{1,2}$

\author{
https://orcid.org/0000-0002-3355-163X \\ https://orcid.org/0000-0002-0461-5673 \\ https://orcid.org/0000-0001-6896-4234 \\ https://orcid.org/0000-0002-9038-9974 \\ https://orcid.org/0000-0002-1878-2237
}

Objetivo: Analisar aspectos epidemiológicos da infecção por COVID-19 nos profissionais de Enfermagem durante a emergência da pandemia no território brasileiro em 2020. Método: Estudo transversal, descritivo, quantitativo e retrospectivo, de profissionais de Enfermagem brasileiros com suspeita e/ou confirmação de infecção por COVID-19 nos meses de março e abril de 2020. Estatística descritiva foi conduzida para os dados sociodemográficos e inferenciais dos dados temporais por meio da modelagem de séries pelo modelo exponencial duplo. Resultados: Houve 8.399 suspeitos, sendo 1.750 confirmados laboratorialmente. A maioria dos profissionais são jovens, do sexo feminino, residentes em todos os Estados, com maior concentração em São Paulo, Rio de Janeiro, Santa Catarina, Ceará, Rio Grande do Sul, Minas Gerais, Pernambuco e Bahia. A atuação profissional predominou em ambiente hospitalar e a distribuição temporal dos casos e óbitos confirmados por COVID-19 apresentou comportamento exponencial. Conclusões: A sensibilização das equipes de Enfermagem quanto a notificação de irregularidades e de casos é um importante recurso para que haja a intensificação de medidas fiscalizatórias e adesão efetiva das medidas preventivas preconizadas, e consequentemente haverá preservação de vidas dos profissionais de Enfermagem e comunidades sob seus cuidados.

Descritores: Epidemiologia; Pandemias; Infecções por Coronavírus; Enfermagem; Profissionais de Enfermagem.

\section{IMPACT OF COVID-19 ON BRAZILIAN NURSING WORK: EPIDEMIOLOGICAL ASPECTS}

Objective: To analyze the epidemiological aspects of COVID-19 infection in nursing professionals during the emergence of the pandemic in Brazil in 2020 . Method: Cross-sectional, descriptive, quantitative and retrospective study of Brazilian nursing professionals with suspected and/or confirmed infection by COVID-19 in the months of March and April 2020. Descriptive statistics was conducted for the sociodemographic and inferential data of the temporal data through the modeling of series by the double exponential model. Results: There were 8,399 suspects, 1,750 of which were laboratory confirmed. Most professionals are young, female, residing in all states, with a greater concentration in São Paulo, Rio de Janeiro, Santa Catarina, Ceará, Rio Grande do Sul, Minas Gerais, Pernambuco and Bahia. Professional performance predominated in the hospital environment and the temporal distribution of cases and deaths confirmed by COVID-19 showed exponential behavior. Conclusions: The awareness of nursing teams regarding the notification of irregularities and cases is an important resource for the intensification of inspection measures and effective adherence to the recommended preventive measures, and consequently there will be preservation of the lives of nursing professionals and communities under their care. care.

Descriptors: Epidemiology; Pandemics; Coronavirus Infections; Nursing; Nursing professionals.

\section{IMPACTO DA COVID-19 EN EL TRABAJO DE ENFERMERÍA BRASILEÑO: ASPECTOS EPIDEMIOLÓGICOS}

Objetivo: analizar los aspectos epidemiológicos de la infección por COVID-19 en profesionales de enfermería durante el surgimiento de la pandemia en Brasil en 2020. Metodo: estudio transversal, descriptivo, cuantitativo y retrospectivo de profesionales de enfermería brasileños con infección sospechada y/o confirmada por COVID-19 en los meses de marzo y abril de 2020. Se realizó estadística descriptiva para los datos sociodemográficos e inferenciales de los datos temporales a través del modelado de series por el modelo exponencial doble. Resultados: Hubo 8.399 sospechosos, 1.750 de los cuales fueron confirmados por laboratorio. La mayoría de los profesionales son jóvenes, mujeres, residentes en todos los estados, con una mayor concentración en São Paulo, Río de Janeiro, Santa Catarina, Ceará, Río Grande del Sur, Minas Gerais, Pernambuco y Bahía. El desempeño profesional predominó en el ambiente hospitalario y la distribución temporal de casos y muertes confirmados por COVID-19 mostró un comportamiento exponencial. Conclusiones: La conciencia de los equipos de enfermería sobre la notificación de irregularidades y casos es un recurso importante para la intensificación de las medidas de inspección y el cumplimiento efectivo de las medidas preventivas recomendadas, y en consecuencia se preservará la vida de los profesionales de enfermería y las comunidades bajo su cuidado. cuidado

Descriptores: Epidemiología; Pandemias; Infecciones por Coronavirus; Enfermería; Profesionales de enfermería.

${ }^{1}$ Universidade do Estado de Mato Grosso, campus de Tangará da Serra, MT.

2 Universidade Federal de Mato Grosso, campus de Cuiabá, MT.

${ }^{3}$ Conselho Federal de Enfermagem, Brasilia, DF.

Autor Correspondente: Ana Cláudia Pereira Terças Trettel. E-mail: ana.claudia@unemat.br Recebido: 09/5/2020 - Aceito: 17/5/2020 


\section{INTRODUÇÃO}

Atualmente, o desafio dos profissionais da saúde por todo o mundo está relacionado a pandemia do novo coronavírus (COVID-19) originada no final de 2019 na China ${ }^{(1)}$. A alta transmissibilidade associada a disseminação por formas de contágio diversas $^{(2)}$ e a inexistência de medicamentos e vacinas eficazes contribuíram para sua expansão pelos continentes, atingindo 4.066.238 casos e 312.845 óbitos no Mundo(3) $^{(3)} 234.106$ casos com 15.662 mortes no Brasil até 17 de maio de $2020^{(4)}$.

Frente a demanda exacerbada pelos serviços de saúde, os enfermeiros ocupam uma posição central, atuando desde a gestão dessa emergência em saúde pública à frentes de prevenção e assistência direta aos acometidos pela COVID-19(5). Essa nova realidade ampliou a vulnerabilidade desses profissionais, pela falta de Equipamentos de Proteção Individual (EPI) disponíveis e possibilidade de infecção durante o cuidado de Enfermagem ${ }^{(6,7)}$

No entanto, pesquisadores ressaltam que o desafio no enfrentamento à COVID-19 perpassa por estoques de EPI limitado, materiais básicos se esgotando e uma demanda crescente de pacientes ${ }^{\left({ }^{(8)}\right.}$. Estudos com enfermeiros que atuam na linha de frente apontaram que a sobrecarga no trabalho reflete claramente na redução do desempenho e possibilidade ampliada à infecção(9-11). Em adicional, é observado no Brasil desatenção e negligência com a equipe de Enfermagem, na imposição de reaproveitamento de EPI e improvisos, profissionais de grupos de risco ou adoecidos impedidos de afastamento e escalas desumanas, quadro que evidencia que a gestão dos serviços de saúde privilegia alguns aspectos, em detrimento da saúde e integridade desses profissionais.

O deslocamento do epicentro para a América ${ }^{(3)}$ e a disseminação da doença no Brasil, trouxe a realidade da pandemia para o cotidiano da Enfermagem brasileira. Assim, problemas históricos como, baixo investimento em infraestrutura, péssimas condições de trabalho ${ }^{(12)} \mathrm{e}$ quantitativo de profissionais insuficientes, especificamente pelo dimensionamento de pessoal aquém do ide$\mathrm{al}^{(13)}$ em meio ao aumento do fluxo de usuários nos serviços de saúde, suspeitos e/ou confirmados com Covid-19, somaram os prejuizos tanto a assistência quanto para saúde desses profissionais.

Embora, mesmo sob riscos constantes de adoecer e com necessidade de valorização e respeito, os profissionais de Enfermagem sustentam um cuidado baseado em evidências científicas que contribui com a saúde da população a nível global(14). E inevitavelmente, não saem ilesos dessa jornada intensa, muitas vezes, vindo a adoecer e/ou a morrer no exercício do cuidar ${ }^{(12)}$. Frente a isso, buscou-se analisar aspectos epidemiológicos da infecção por COVID-19 nos profissionais de Enfermagem durante a emergência da pandemia no território brasileiro em 2020.

\section{MÉTODO}

\section{Tipo de estudo}

Estudo transversal, retrospectivo e quantitativo, com dados secundários e de domínio público.

\section{Local do Estudo}

Realizado em todo o território nacional brasileiro, a partir das notificações realizadas nos serviços públicos e privados que possuem profissionais de Enfermagem registrados nos respectivos Conselhos Regionais de Enfermagem (Coren).

\section{Participantes do Estudo}

As notificações correspondem ao total de 8.399 profissionais de Enfermagem. Teve como critério de inclusão, notificações de Auxiliares de Enfermagem, Técnicos de Enfermagem e Enfermeiros, com suspeita clínica ou confirmação de infecção por COVID-19 de 23 de março (data do primeiro registro) a 28 de abril de 2020. Estabeleceu-se como critério de exclusão, os registros que não possuiam informações relacionadas ao sexo, idade e estado de residência.

\section{Procedimentos de Coleta de dados}

A coleta de dados ocorreu na primeira semana de maio de 2020. Para a realização deste trabalho, utilizou-se os boletins do Observatório de Enfermagem, disponibilizados pelo Conselho Federal de Enfermagem (Cofen) (http://observatoriodaenfermagem.cofen.gov.br/), em relação aos casos suspeitos, confirmados e óbitos de profissionais de Enfermagem por COVID-19 no país. As variáveis utilizadas foram: número total diário de profissionais de Enfermagem com suspeita da COVID-19 e em quarentena, hospitalizados ou que foram a óbito; número total diário de profissionais de Enfermagem confirmados por COVID-19 em quarentena, hospitalizados ou que foram a óbito; sexo, idade, local de trabalho e estado de residência.

\section{Procedimentos de análise e tratamento dos dados}

As informações foram analisadas por meio dos programas SPSS® versão 20.0 e MINITAB® versão 17. Os dados quantitativos foram analisados utilizando técnicas estatísticas descritivas e inferenciais. Descritivamente por meio de distribuição de frequências, proporções, médias e desvio padrão apresentados em tabelas. A seguir, na análise inferencial, foi utilizada a técnica estatística de séries temporais, considerando o modelo exponencial duplo e também foram utilizados mapas construídos pelo observatório COFEN por meio do $\operatorname{ArcGIS}^{\circledR}$. 


\section{Aspectos éticos}

Este estudo respeitou todos os aspectos éticos de pesquisa, conforme as diretrizes da Resolução № 466/2012 do Conselho Nacional de Saúde (CNS), Não sendo necessário parecer de Comitê de Ética por utilizar dados de domínio público.

\section{RESULTADOS}

Dos 8.399 profissionais de Enfermagem com suspeita da COVID-19 no Brasil entre os meses de março e abril de 2020, 1.750 foram confirmados laboratorialmente, sendo importante ressaltar que os demais aguardam resultado de exame confirmatório em decorrência da morosidade na liberação dos laudos. Em relação as características sociodemográficas e profissionais, a população geral desse estudo apresentou idade média de 38,36 anos e um desvio padrão de 8,55; a maioria era do sexo feminino (77,49\%), com faixa etária entre 35 e 59 anos (67,83\%) (Tabela 1). Residentes nos Estados do Rio de Janeiro (29,37\%) e São Paulo (27,89\%) (Figura 1). O local de atuação profissional prevaleceu o ambiente hospitalar (71,54\%) (Tabela 2). Ainda na Tabela 1, é possivel observar que a maioria se encontra em isolamento domiciliar. Destaca-se também, o número elevado de profissionais com suspeita da COVID-19 hospitalizados ( $n=173$ ) e que evoluíram para óbito $(\mathrm{N}=15)$ sem diagnóstico laboratorial.

Tabela 1 - Distribuição por sexo e faixa etária dos casos suspeitos e confirmados de profissionais de Enfermagem por COVID-19 no Brasil. Março e abril de 2020.

\begin{tabular}{|c|c|c|c|c|c|c|}
\hline \multirow{2}{*}{$\begin{array}{c}\text { Situação dos profissionais de } \\
\text { Enfermagem }\end{array}$} & \multicolumn{2}{|c|}{ Sexo } & \multicolumn{2}{c|}{ Faixa etária (anos) } & \multirow{2}{*}{ Total } \\
\cline { 2 - 6 } & $\begin{array}{c}\text { Femi- } \\
\text { nino }\end{array}$ & $\begin{array}{c}\text { Mas- } \\
\text { culino }\end{array}$ & $18-34$ & $35-59$ & $>60$ & \\
\hline $\begin{array}{c}\text { Suspeita da COVID-19 em } \\
\text { isolamento domiciliar }\end{array}$ & 5445 & 1016 & 1861 & 4519 & 81 & 6461 \\
\hline $\begin{array}{c}\text { Óbito por suspeita da CO- } \\
\text { VID-19 }\end{array}$ & 8 & 7 & 0 & 12 & 3 & 15 \\
\hline $\begin{array}{c}\text { Suspeita da COVID-19 inter- } \\
\text { nados }\end{array}$ & 135 & 38 & 25 & 142 & 6 & 173 \\
\hline $\begin{array}{c}\text { Confirmado para COVID-19 em } \\
\text { isolamento domiciliar }\end{array}$ & 1319 & 366 & 528 & 1139 & 18 & 1685 \\
\hline $\begin{array}{c}\text { Confirmado para COVID-19 } \\
\text { internados }\end{array}$ & 8 & 7 & 4 & 11 & 0 & 15 \\
\hline $\begin{array}{c}\text { Óbito confirmado para CO- } \\
\text { VID-19 falecidos }\end{array}$ & 29 & 21 & 3 & 37 & 10 & 50 \\
\hline \begin{tabular}{c} 
Total \\
\hline
\end{tabular} & 6944 & 1455 & 2421 & 5860 & 118 & 8399 \\
\hline
\end{tabular}

Fonte: Tabela gerada a partir dos dados do observatório de Enfermagem do COFEN, 2020 - http://observatoriodaenfermagem.cofen.gov.br/.
Houve maior frequência de notificações de profissionais atuando em ambiente hospitalar, embora há registros de vários contextos laborais da Enfermagem, inclusive em atividades que não incluem exclusivamente o contato direto com o paciente, como na fiscalização de Conselhos de Classe.

Tabela 2 - Distribuição por local de atuação dos profissionais de Enfermagem suspeitos e confirmados da COVID-19 no Brasil. Março e abril de 2020.

\begin{tabular}{|c|c|c|c|c|c|c|c|}
\hline \multirow[b]{2}{*}{\begin{tabular}{|c|} 
Local de \\
atuação \\
profissio- \\
nal
\end{tabular}} & \multicolumn{6}{|c|}{ Situação } & \multirow[b]{2}{*}{ Total } \\
\hline & $\begin{array}{c}\text { Suspeita } \\
\text { da CO- } \\
\text { VID-19 } \\
\text { em qua- } \\
\text { rentena }\end{array}$ & $\begin{array}{c}\text { Suspeita } \\
\text { da CO-- } \\
\text { VID-19 } \\
\text { faleci- } \\
\text { dos }\end{array}$ & $\begin{array}{c}\text { Suspeita } \\
\text { da CO- } \\
\text { VID-19 } \\
\text { interna- } \\
\text { dos }\end{array}$ & \begin{tabular}{|} 
Confir- \\
mado \\
para \\
CO- \\
VID-19 \\
em \\
quaren- \\
tena
\end{tabular} & $\begin{array}{c}\text { Confir- } \\
\text { mado } \\
\text { para } \\
\text { CO- } \\
\text { VID-19 } \\
\text { interna- } \\
\text { dos }\end{array}$ & $\begin{array}{c}\text { Confir- } \\
\text { mado } \\
\text { para } \\
\text { CO- } \\
\text { VID-19 } \\
\text { faleci- } \\
\text { dos }\end{array}$ & \\
\hline $\begin{array}{l}\text { Ambula- } \\
\text { tório }\end{array}$ & 7 & 0 & 1 & 8 & 0 & 0 & 16 \\
\hline Asilo & 137 & 0 & 1 & 27 & 0 & 0 & 165 \\
\hline $\begin{array}{l}\text { Atenção } \\
\text { Primária }\end{array}$ & 129 & 0 & 6 & 38 & 0 & 0 & 173 \\
\hline CAPS & 8 & 0 & 1 & 5 & 0 & 0 & 14 \\
\hline $\begin{array}{c}\text { Companhia } \\
\text { Operadora } \\
\text { de Rodo- } \\
\text { vias }\end{array}$ & 0 & 0 & 0 & 1 & 0 & 0 & 1 \\
\hline $\begin{array}{l}\text { Fiscal } \\
\text { Coren }\end{array}$ & 0 & 0 & 0 & 2 & 0 & 0 & 2 \\
\hline $\begin{array}{c}\text { Hemocen- } \\
\text { tro }\end{array}$ & 7 & 0 & 1 & 8 & 0 & 0 & 16 \\
\hline $\begin{array}{l}\text { Hemodi- } \\
\text { álise }\end{array}$ & 0 & 0 & 0 & 2 & 0 & 0 & 2 \\
\hline $\begin{array}{l}\text { Hospital } \\
\text { Psiquiá- } \\
\text { trico }\end{array}$ & 0 & 0 & 0 & 3 & 0 & 0 & 3 \\
\hline Hospital & 4813 & 14 & 119 & 1252 & 12 & 43 & 6253 \\
\hline $\begin{array}{l}\text { Pronto } \\
\text { Atendi- } \\
\text { mento }\end{array}$ & 1353 & 1 & 43 & 333 & 3 & 7 & 1740 \\
\hline SAMU & 7 & 0 & 1 & 6 & 0 & 0 & 14 \\
\hline Total & 6461 & 15 & 173 & 1685 & 15 & 50 & 8399 \\
\hline
\end{tabular}

Fonte: Tabela gerada a partir dos dados do observatório de Enfermagem do COFEN, 2020 - http://observatoriodaenfermagem.cofen.gov.br/.

Houve registro em todos os Estados brasileiros, porém a maior concentração de casos suspeitos/confirmados da COVID-19 nos profissionais de Enfermagem está em São Paulo (2.438) e Rio de Janeiro (2.056), porém Santa Catarina (661), Ceará (536), Rio Grande do Sul (383), Minas Gerais (372), Pernambuco (259) e Bahia 
(273) apresentaram registros superiores a 200 casos. Em se tratando de óbitos São Paulo, Rio de Janeiro e Ceará se destacam. O vírus vem ocupando diversas regiões do país, logo transitando em variados tipos de climas e biomas.

Figura 1 - Distribuição por estado da federação dos profissionais de Enfermagem suspeitos e confirmados da COVID-19 no Brasil. Março e abril de 2020.

\section{Distribuição de casos dos profissionais de enfermagem suspeitos/confirmados para COVID-19 no Brasil}

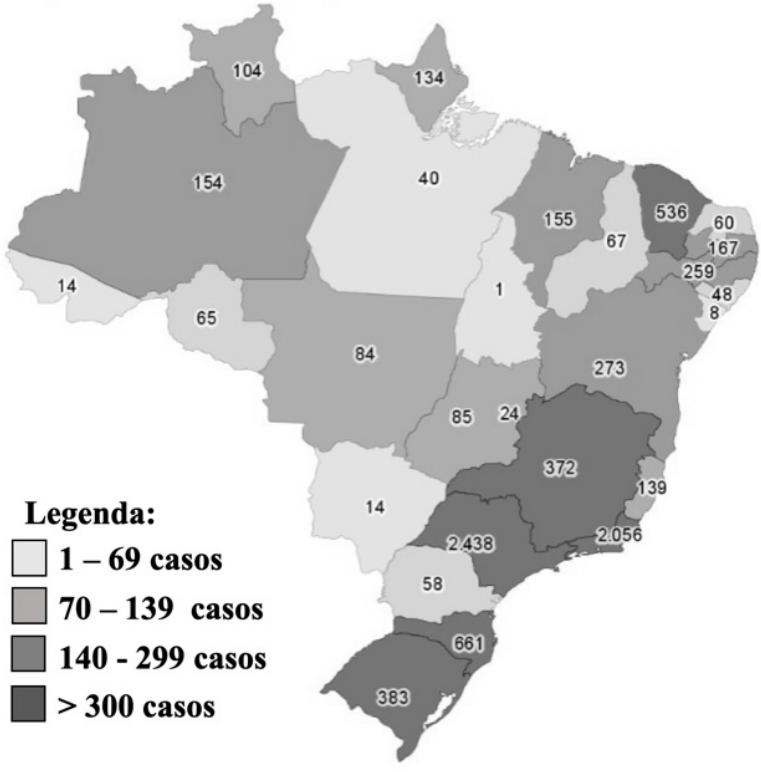

Distribuição de óbitos dos profissionais de enfermagem suspeitos/confirmados por COVID-19 no Brasil

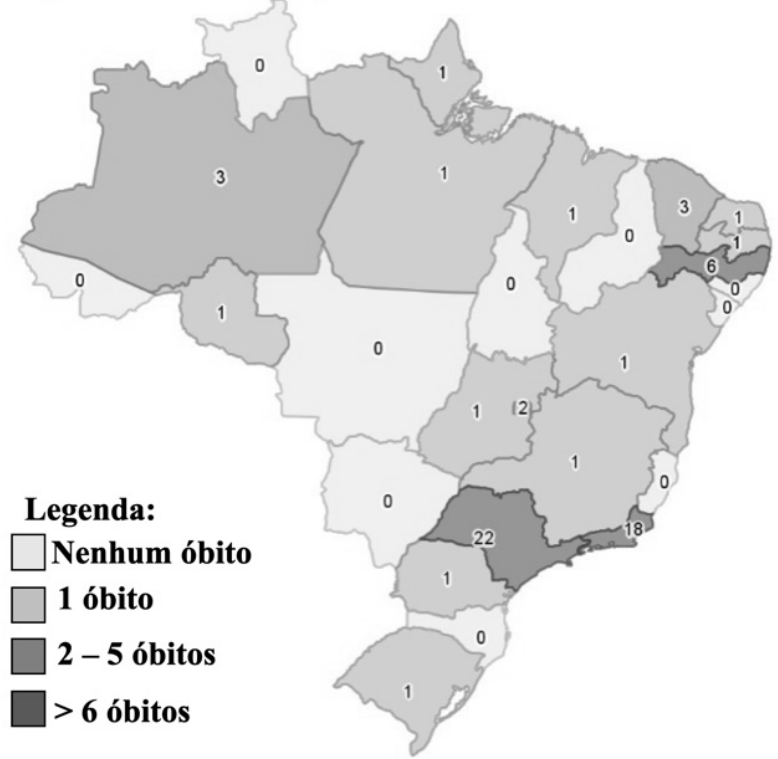

Fonte: Mapas gerados a partir dos dados do observatório de Enfermagem do COFEN, 2020 - http://observatoriodaenfermagem.cofen.gov.br/.
Com relação a distribuição temporal dos casos e óbitos confirmados por COVID-19 dos profissionais de Enfermagem no Brasil, o comportamento exponencial é evidenciado nas Figuras 2 e 3, o que remete a um crescimento progressivo e ascendente. Observa-se também que esse comportamento indica o grau de exposição e vulnerabilidade desses profissionais para o contágio e óbito.

Figura 2 - Gráfico do número de casos e óbitos confirmados e acumulados dos profissionais de Enfermagem suspeitos e confirmados por COVID-19 no Brasil com valores ajustados pelo modelo exponencial simples.
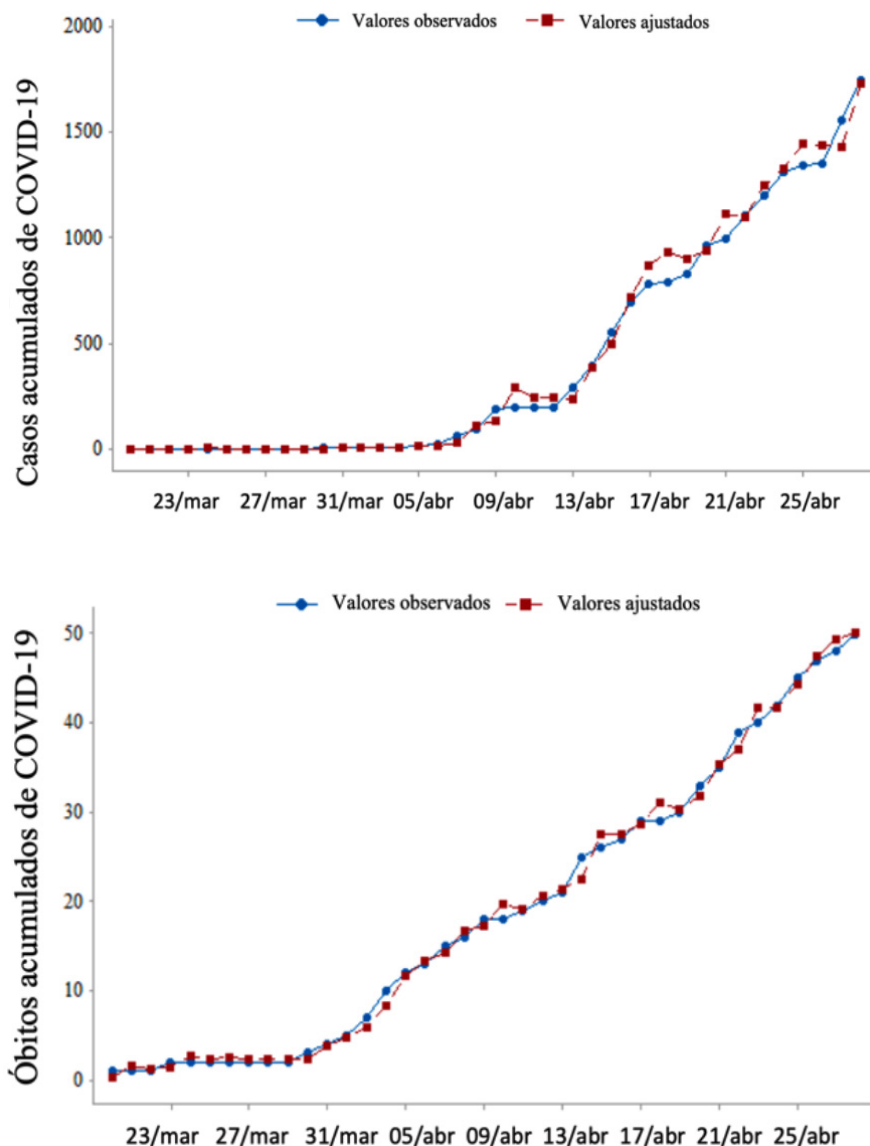

Fonte: Mapas gerados a partir dos dados do observatório de Enfermagem do COFEN, 2020 - http://observatoriodaenfermagem.cofen.gov.br/

Na Figura 2, observa-se um comportamento exponencial dos números de casos (valores reais) e ajustados pelo modelo exponencial duplo, o mesmo em relação aos óbitos. Cabe destacar que após a análise residual, foi necessária uma transformação nos dados. Nesta situação, a transformação indicada pelo método de Box e Cox ${ }^{(19)}$, para os casos 
foi dos casos elevados a 0,1425179406 $\left(\mathrm{y}^{*}=\mathrm{y}^{(0,1425179406)}\right)$ e para os óbitos foi dos casos elevados a 0,50 (y = y).

Os valores de alfa e gama, ajustados pelo modelo exponencial duplo para o número de casos da COVID-19 no Brasil dos dados foram 1,19790 (a = 1,19790) e 0,19601 ( $g=0,19601)$ para alfa e gama, respectivamente o valor da $E M A=2,55961 \%$. Já para os óbitos foram de 1,25094 $(\mathrm{a}=1,25094)$ e 0,01000 ( $\mathrm{g}=0,01000)$, respectivamente para alfa e gama e o valor da $E M A=6,23 \%$.

\section{DISCUSSÃO}

Entre os aspectos epidemiológicos, verificou-se o acometimento de profissionais na faixa etária produtiva, o que aponta um impacto direto da pandemia na redução da força de trabalho da Enfermagem brasileira. Estudo em Henan confirma esses achados ao identificar que a faixa etária de casos por COVID-19 se mantem no período em que as pessoas estão mais ativas em seus contextos laborais ${ }^{(15)}$. Além disso, essa infecção nesse momento mostra preferência tanto por idade (fora dos grupos de risco) como por gênero específico ${ }^{(16)}$, também em concordância com o estudo.

Durante a pandemia, os profissionais de Enfermagem se veem responsáveis pelo bem-estar dos pacientes, assumindo o dever em meio a um contexto totalmente novo, sem, no entanto, a experiência para lidar com esse cenário, e são nesses momentos que se mostram mais vulneráveis à infecção e transmissão(17). Os profissionais de enfermagem estão diretamente expostos aos riscos de infecção por COVID-19, e estudo chinês indica que o contágio no contexto laboral desses profissionais, ocorre não somente por falta/inadequação de EPI, mas pelo desconhecimento da testagem positiva de pares e pessoas da comunidade, e medidas de gerenciamento de ris$\mathrm{co}^{(18)}$. Esse aspecto também foi observado nesse estudo, especificamente ao vislumbrar número significativo de casos suspeitos e notificações em ambientes e/ou atividades consideradas de menor risco; uma particularidade da COVID-19, por estar presente em locais diversos, inclusive naqueles ambientes tidos como não tradicionais para o cuidado intensivo de Enfermagem(19).

Outro motivo para esses profissionais estarem cotidianamente expostos e adoecerem por COVID-19, ocorre porque a Enfermagem possui responsabilidades únicas no cuidar em saúde, independente do cenário existente ${ }^{(20-21)}$, e a assistência que presta envolve não somente o contato direto com o paciente, mas a interação com o ambiente, onde superfícies, objetos e EPI podem estar contaminados pelo vírus ${ }^{(22)}$

A distribuição geográfica das infecções nesses profissio- nais foi mais incidente em dois grandes centros populacionais (São Paulo e Rio de Janeiro), que possuem os maiores aeroportos internacionais, bem como elevadas densidades populacionais, de serviços de saúde e de profissionais. Sendo esses os locais onde a doença emergiu, como foi visto no inicio da pandemia em Wuhan ${ }^{(1)}$, no epicentro europeu na Itália ${ }^{(23)}$ e mais recentemente no americano - Estados Unidos ${ }^{(3)}$.

Ademais, a infecção da maioria dos pacientes com SARS-cov e MERS em surtos anteriores de coronavírus se deu em estabelecimentos de saúde ${ }^{(24)}$, mantendo comportamento semelhante com a pandemia atual ${ }^{(25)}$. Todavia, em novos territórios a expansão da COVID-19 ocorreu inicialmente por casos importados assintomáticos ${ }^{(26)}$, que se infectaram em ambientes de saúde ${ }^{(18)}$ e familiares ${ }^{(27)}$. Como os profissionais de Enfermagem compõem o maior quantitativo nas frentes de assistência à saúde, tornamse vulneráveis a infecção logo no início da doença em seu local de trabalho ${ }^{(28)}$, como demonstrado pela distribuição geográfica dos casos brasileiros.

À medida que o surto da COVID-19 progride, o acompanhamento diário do comportamento epidemiológico assume papel decisivo no direcionamento das ações em saúde mais assertivas ${ }^{(29)}$. Experiências internacionais já apontam a efetividade da utilização de observatórios epidemiológicos durante a pandemia ${ }^{(30,31)}$, embora na América do Sul, o Brasil esteja sendo pioneiro ao monitorar a incidência dos casos entre os profissionais de Enfermagem.

Esse monitoramento vem acionando as instituições e sociedade diante do impacto negativo da pandemia junto à Enfermagem e frente a constatação de evolução exponencial dos casos para outros Estados do Nordeste, Norte e Sul. Por consequência, há redução progressiva dos profissionais de Enfermagem, seja por afastamento, por integrarem grupo de risco, por suspeita/confirmação ou óbitos, que comprometem as estratégicas de enfrentamento à COVID-19(32), além dos processos de sofrimento mental e luto implicados ${ }^{(12)}$.

Estudo coreano ressalta os impactos à saúde mental daqueles que permanecem nas frentes de trabalho, o que reflete em consequências muitas vezes invisibilizadas, mas que causam ansiedade, estresse, pânico, problemas na comunicação, faltas injustificadas e aumento de erros laborais, este último particularmente, sendo uma das principais causas reconhecidas na transmissão do vírus para os profissionais de enfermagem ${ }^{(33)}$

Anteriormente a pandemia da COVID-19, Enfermeiros brasileiros destacaram que a qualidade de vida é polissêmica e relacionada diretamente ao trabalho ${ }^{(34)}$, sendo vivenciada historicamente de forma fragmentada, com baixo bem estar no labore, má remuneração, sobrecarga de trabalho e falta 
de materiais/estrutura ${ }^{35}$. Há ainda problemas de empregabilidade plena, denunciando o grave problema de desequilibrio entre oferta e demanda desta força de trabalho(21).

Esses aspectos são impulsionados nesse momento de pandemia, especialmente em regiões com problemas de maior desigualdade social e no quantitativo/distribuição de profissionais e suprimentos ${ }^{(36)}$, tendo como resultados, mais adoecidos e óbitos crescentes. Há previsão de ápice da curva de casos no mês de maio, o que coincide com as comemorações da profissão no país e no ano eleito pela Organização Mundial da Saúde (OMS) como o "Ano Internacional dos Enfermeiros e Parteiras", por conta do bicentenário de nascimento de Florence Nightingale, para a valorização desses profissionais. O ano de 2020 está sendo um marco histórico de luta, resistência e força da Enfermagem Mundial.

\section{Limitações do estudo}

Entre as limitações do estudo, destaca-se a utilização de dados secundários, que sofrem influência pela incompletude no preenchimento das notificações e possíveis subnotificações.

\section{Contribuições do estudo para a prática}

O conhecimento sobre os casos de adoecimento por COVID-19 nos profissionais de Enfermagem do Brasil, permite reconhecer a vulnerabilidade desses profissionais nos diversos ambientes de cuidado, apontando a necessidade urgente de estratégias que minimizem os riscos de infecção e a permanência desses danos, que comprometem a saúde/vida do trabalhador.

\section{CONCLUSÃO}

Após 55 dias do registro do primeiro profissional de Enfermagem com COVID-19 no Brasil, há número cres- cente de casos suspeitos, confirmados e óbitos. Na maioria, são profissionais jovens, do sexo feminino, residentes principalmente na região sudeste, porém com registros em expansão para região Norte, Nordeste e Sul. A atuação profissional predominou em ambiente hospitalar e a distribuição temporal dos casos e óbitos confirmados por COVID-19 apresentou comportamento exponencial.

Esses aspectos demonstram que a pandemia da $\mathrm{CO}-$ VID-19 no Brasil não está isolada em determinadas regiões, tampouco que os profissionais de Enfermagem estejam inteiramente assistidos pelas instituições e gestores. A sensibilização das equipes quanto a notificação de irregularidades e de casos é um importante recurso para que haja a intensificação de fiscalizações e adesão efetiva das medidas preventivas preconizadas, consequentemente vidas serão poupadas, tanto dos profissionais como da população que necessita do cuidado em saúde.

Não obstante, há necessidade de novas políticas públicas que sejam direcionadas às necessidades dos profissionais de Enfermagem, contemplando as condições de trabalho, jornadas, carreira, piso salarial e disposição de recursos suficientes para o exercício profissional. Tais políticas devem garantir amparo aos profissionais e potencializar o cuidado em saúde, ao passo, que valorize e proteja o trabalhador, sua saúde, a qualidade da assistência e o fortalecimento da ciência de Enfermagem.

\section{Contribuições dos autores}

Vagner Ferreira do Nascimento, Mariano Martinez Espinosa, Manoel Carlos Neri da Silva, Neyson Pinheiro Freire e Ana Cláudia Pereira Terças Trettel contribuíram em todas as etapas, ou seja, concepção e/ou desenho do estudo, coleta, análise e interpretação dos dados, redação e revisão crítica do manuscrito e aprovação da versão final a ser publicada.

\section{REFERÊNCIAS}

1. Wang C, Horby PW, Hayden FG, Gao GF. A novel coronavirus outbreak of global health concern. Lancet. [internet]. 2020 [cited 2020 apr 29]; 395(10223):470-473. Available from: https://www.thelancet.com/journals/lancet/article/PIISO140-6736(20)30185-9/fulltext https://doi. org/10.1016/S0140-6736(20)30185-9.

2. Li-sheng W, Yi-ru W, Da-wei Y, Qing-quan L. A review of the 2019 Novel Coronavirus (COVID-19) based on current evidence, Inter J Antimicrob Agents. [internet]. 2020 [cited 2020 apr 29]; 105948. Available from: https://www.sciencedirect. com/science/article/pii/S0924857920300984. https://doi.or-

\section{g/10.1016/j.ijantimicag.2020.105948}

3. World Health Organization (WHO). Coronavirus disease 2019 (COVID-19) SituationReport-67. [internet]. 2020. [cited 2020 apr 27]: Available from: https://www.who.int/emergencies/diseases/novel-coronavirus-2019/situation-reports/.

4. Ministério da Saúde (BR). Boletim Epidemiológico № 05. Centro de operações de emergência em Saúde Pública - COVID-19. Brasilia: Ministério da Saúde: 2020. . [internet]. 2020. [cited 2020 apr 27]. Available from: https://www.saude.gov.br/images/pdf/2020/ April/10/10.04.2020-COVID.pdf. 
5. Sue AB, Petra B. 2020 year of the nurse and midwife: Meeting new challenges. Int Emerg Nurs. [internet]. 2020 [cited 2020 apr 29]; 49:100848. Available from: https://www.sciencedirect.com/science/ article/pii/S1755599X20300203?via\%3Dihub https://doi.org/10.1016/j. ienj.2020.100848.

6. Guangming Y, Hualiang L, Liangjun C, Wang S, Zeng Z, Wang W, et al. Environmental contamination of the SARS-CoV-2 in healthcare premises: An urgent call for protection for healthcare workers. medRXIV. [internet]. 2020 [cited 2020 apr 29]; 1-20. Available from: https:// www.medrxiv.org/content/10.1101/2020.03.11.20034546vl.full.pdf+html https://doi.org/10.1101/2020.03.11.20034546.

7. Klement E, Godefroy N. Burrel S, Kornblum D, Monsel G, Bleibtreu A, et al. The first locally acquired novel case of 2019-nCoV infection in a healthcare worker in the Paris área. Clin Infect Dis. [internet]. 2020 [cited 2020 apr 29]; ciaal71:1-4. Available from: https://academic.oup.com/ cid/article/doi/10.1093/cid/ciaal71/5811445. https://doi.org/10.1093/ cid/ciaal71.

8. Ling L, Joynt GM, Lipman J, Constantin JM, Joannes-Boyau O. COVID-19: A critical care perspective informed by lessons learnt from other viral epidemics. Anaesth Crit Care PA. [internet]. 2020 [cited 2020 apr 29]; 39(2):163-166. Available from: https://www.sciencedirect. com/science/article/pii/S2352556820300291?via\%3Dihub. https:// doi.org/10.1016/j.accpm.2020.02.002.

9. Liu Y, Wang H, Chen J, Zhang X, Yue X, Ke J, et al. Emergency management of nursing human resources and supplies to respond to coronavirus disease 2019 epidemic. Inter J Nur Scie. [internet]. 2020 [cited 2020 apr 29]; 1-4. Available from: https://www.sciencedirect.com/ science/article/pii/S235201322030051X. https://doi.org/10.1016/j. ijnss.2020.03.011.

10. Kang L, Ma S, Chen M, Yang J, Wang Y, Li R, et al. Impact on mental health and perceptions of psychological care among medical and nursing staff in Wuhan during the 2019 novel coronavirus disease outbreak: A cross-sectional study. Brain Behav Immun. [internet]. 2020 [cited 2020 apr 29]; 1-7. Available from: https://www.sciencedirect. com/science/article/pii/S0889159120303482?via\%3Dihub. https://doi. org/10.1016/j.bbi.2020.03.028.

11. Jiang L, Broomer ME, Ning C. The performance and professionalism of nurses in the fight against the new outbreak of COVID-19 epidemic is laudable. Inter J Nurs Stud. [internet]. 2020 [cited 2020 apr 29]; 103578. Available from: https://www.sciencedirect.com/science/article/pii/S0020748920300638?via\%3Dihub. https://doi.org/10.1016/j. ijnurstu.2020.103578.

12. Tavares CQ. Dimensões do cuidado na perspectiva da espiritualidade durante a pandemia pelo novo coronavirus (COVID-19). J Health NPEPS. [Internet]. 2020 [cited 2020 apr 29]; 5(1):1-4. Available from: https://periodicos.unemat.br/index.php/jhnpeps/article/view/4517. http://dx.doi.org/10.30681/252610104517.

13. Lopes FG, Palotti PLM, Barbosa SCT, Massaco KN. Nota Técnica n. 30 (Diest): Mapeamento dos profissionais de saúde no Brasil: alguns apontamentos em vista da crise sanitária da COVID-19. Available from: http://repositorio.ipea.gov.br/handle/11058/9837.

14. The Lancet. 2020: unleashing the full potential of nursing. Lancet. [internet]. 2019 [cited 2020 apr 29]; 394(10212):p1879. Available from: https://www.thelancet.com/journals/lancet/article/ PIIS0140-6736(19)32794-1/fulltext. https://doi.org/10.1016/S01406736(19)32794-1.

15. Wang P. Lu J, Jin Y, Zhu M, Wang L, Chen S. Statistical and network analysis of 1212 COVID-19 patients in Henan, China. Int $\mathrm{J}$ Infect Dis. [internet]. 2020 [cited 2020 apr 29]. Available from: https://www.ncbi. nlm.nih.gov/pmc/articles/PMC7180361/. https://doi.org/10.1016/j. ijid.2020.04.051.

16. Chen P. Zhang Y, Wen Y, Guo J, Jia J, Ma Y, et al. Epidemiological and clinical characteristics of 136 cases of COVID-19 in main district of Chongqing. J Formos Med Assoc. [internet]. 2020 [cited 2020 apr 29]. Available from: https://www.sciencedirect.com/science/article/ pii/S0929664620301558\#!. https://doi.org/10.1016/j.jfma.2020.04.019.

17. Liu Q, Luo D, Haase JE, Guo Q, Wang XQ, Liu S, et al. The experiences of health-care providers during the COVID-19 crisis in China: a qualitative study. Lancet. [internet]. 2020 [cited 2020 apr 29]. Available from: https://www.thelancet.com/journals/langlo/article/ PIIS2214-109X(20)30204-7/fulltext. https://doi.org/10.1016/S2214$109 \times(20) 30204-7$.

18. Xiao SW, Xiao XR, Zhang JC, Yang WB, Ma WL, Yang BH. A cluster of health care workers with COVID-19 pneumonia caused by SARSCoV-2. J microbiol immunol infect. [internet]. 2020 [cited 2020 apr 29]. Available from: https://www.sciencedirect.com/science/article/pii/ S1684118220301079. https://doi.org/10.1016/j.jmii.2020.04.013.

19. Jennings BM, Yager KA. From Fear to Fortitude: Using the Power Within the Nursing Profession to Fight COVID-19. Nursing Outlook. [internet]. 2020 [cited 2020 apr 18]. Available from: https://www.nursingoutlook.org/article/S0029-6554(20)30248-7/fulltext. https://doi. org/10.1016/j.outlook.2020.04.008.

20. Choi KR, Skrine Jeffers K, Logsdon, CM. Nursing and the novel coronavirus: Risks and responsibilities in a global outbreak. J Adv Nurs. [internet]. 2020 [cited 2020 apr 29]. Available from: https://www.ncbi. nlm.nih.gov/pubmed/32202336. https://doi.org/10.1111/jan.14369.

21. Machado MH, Oliveira E, Lemos W, Lacerda WF, Aguiar Filho W, Wermelinger $M$, et al. Mercado de trabalho da enfermagem: aspectos gerais. Enferm Foco. [internet]. 2016 [cited 2020 apr 29]: 
7(ESP):35-62. Available from: http://revista.cofen.gov.br/index.php/ enfermagem/article/view/691/301. https://doi.org/10.21675/2357707X.2016.v7.nESP.691.

22. Ye G, Lin H, Chen L, Wang S, Zeng Z, Wang W, et al. Environmental Contamination of SARS-CoV-2 in Healthcare Premises. J Infect. [internet]. 2020 [cited 2020 apr 29]. Available from: https://www.journalofinfection.com/article/S0163-4453(20):30260-7/pdf. https://doi. org/10.1016/j.jinf.2020.04.034.

23. Spina S, Marrazzo F, Migliari M, Stucchi, Forza A, Fumagali R. The response of Milan's Emergency Medical System to the COVID-19 outbreak in Italy. 2020. Lancet. [Internet]. 2020 [cited 2020 May 3]; 395(10227):e49-e50. Available from: https://www.thelancet.com/journals/lancet/article/PIISO140-6736(20)30493-1/fulltext.

24. Chowell G, Abdirizak F, Lee S, Lee J, Jung E, Nishiura H, et al. Transmission characteristics of MERS and SARS in the healthcare setting: a comparative study. BMC Med. [Internet]. 2015 [cited 2020 May 3]; 13:210. Available from: https://www.ncbi.nlm.nih.gov/pmc/articles/ PMC4558759/. http://dx.doi.org/10.1186/s12916-015-0450-0.

25. Haider N, Yavlinsky A, Simons D, Osman AY, Ntoumi F, et al. Passengers' destinations from China: low risk of Novel Coronavirus (2019-nCoV) transmission into Africa and South America. Epid infect. [Internet]. 2020 [cited 2020 May 3]; 148:e41. Available from: https://www.cambridge.org/core/journals/epidemiology-and-infection/article/passengers-destinations-from-china-low-risk-of-novel-coronavirus-2019ncov-transmission-into-africa-and-south-america/886D3CABC0ED422A511086DDB836B34F. https://doi.org/10.1017/ S0950268820000424.

26. Zhou P, Yang X-L, Wang X-G, Hu B, Zhang L, Zhang W, et al. A pneumonia outbreak associated with a new coronavirus of probable bat origin. Nature. [Internet]. 2020 [citado 2020 apr 30]; 579:270-273. Available from: https://www.biorxiv.org/content/10.1101/2020.01.22.914952v2 https://doi.org/0.1038/s41586-020-2012-7.

27. Li C, Ji F, Wang L, Wang L, Hao J, Dai M, et al. Asymptomatic and human-to-human transmission of SARS-CoV-2 in a 2-family cluster, Xuzhou, China. Emerg Infect Dis. [Internet]. 2020 [citado 2020 apr 30]; 26(7). Available from: https://wwwnc.cdc.gov/eid/article/26/7/20-0718_ article. https://doi.org/10.3201/eid2607.200718.

28. Rothe C, Schunk M, Sothmann P. Bretzel G, Froeschl G, Wallrauch C, et al. Transmission of 2019-nCoV infection from an asymptomatic contact in Germany. N Engl J Med. [Internet]. 2020 [citado 2020 apr 30] 382:970-971. Available from: https://www.nejm.org/doi/full/10.1056/ NEJMc2001468. https://doi.org/10.1056/NEJMc2001468.

29. Sun K, Chen J, Viboud C. Early epidemiological analysis of the coronavirus disease 2019 outbreak based on crowdsourced data: a population-level observational study. Lancet. [internet]. 2020 [cited 2020 apr
29]. 2(4):e201-e208. Available from: https://www.thelancet.com/journals/landig/article/PIIS2589-7500(20)30026-1/fulltext. https://doi. org/10.1016/S2589-7500(20)30026-1.

30. Tang B, Wang X, Li Q, Bragazzi NL, Tang S, Xiao Y, et al. Estimation of the Transmission Risk of the 2019-nCoV and Its Implication for Public Health Interventions. J Clin Med. [Internet]. 2020 [citado 2020 apr 30]; 9(2). Available from: https://www.mdpi.com/2077-0383/9/2/462 https://doi.org/10.3390/jcm9020462

31. Roosa K, Lee Y, Luo R, Kirpich A, Rothenberg R, Hyman JM, et al. Real-time forecasts of the 261 COVID-19 epidemic in China from February 5th to February 24th, 2020. Infect Dis Modell. [Internet]. 2020 [citado 2020 apr 30]: 5:256-563. Available from: https://www.sciencedirect. com/science/article/pii/S2468042720300051?via\%3Dihub https://doi. org/10.1016/j.idm.2020.02.002.

32. Wang D, Hu B, Hu C, Zhu F, Liu X, Zhang J, et al. Clinical characteristics of 138 hospitalized patients with 2019 novel coronavirus-infected pneumonia in Wuhan, China. J Am Med Assoc. [Internet]. 2019 [citado 2020 apr 30]; 323(11):1061-1069. Available from: https://jamanetwork.com/journals/jama/fullarticle/2761044 https://doi.org/10.1001/ jama.2020.1585

33. Son H, Lee W, Kim H, Lee K, You M. Examination of Hospital Workers' Emotional Responses to an Infectious Disease Outbreak: Lessons From the 2015 MERS Co-V Outbreak in South Korea. Disaster Med Public Health Prep. [Internet]. 2019 [citado 2020 apr 30]; 13(3):504510. Available from: https://www.cambridge.org/core/journals/disaster-medicine-and-public-health-preparedness/article/examination-of-hospital-workers-emotional-responses-to-an-infectiousdisease-outbreak-lessons-from-the-2015-mers-cov-outbreak-insouth-korea/F9C095D8D5AD810C067A2A2CAAD7FAB2 https://doi. org/10.1017/dmp.2018.95

34. Santos DAC, Morais DSV, Franco RVB, Gomes JRAA. Qualidade de vida sob a ótica de enfermeiros de Centro Cirúrgico de um hospital público. Rev Enferm foco. [internet]. 2019 [cited 2020 apr 29];10(4):7-11. Available from: http://revista.cofen.gov.br/index.php/enfermagem/article/view/1676/596.

35. Lopes CCP, Ribeiro TP, Martinho NJ. Sindrome de Burnout e sua relação com a ausência de qualidade de vida no trabalho do enfermeiro. Rev Enferm foco. [internet]. 2012 [cited 2020 apr 29]; 3(2):97-101. Available from: http://revista.cofen.gov.br/index.php/enfer-magem/article/ view/264/151.

36. Mendonça FD, Rocha SS, Pinheiro DLP, Oliveira SV. Região Norte do Brasil e a pandemia da COVID-19: análise socioeconômica e epidemiológica. J Health NPEPS. [internet]. 2020 [cited 2020 apr 29]; 5(1):20-37. Available from: https://periodicos.unemat.br/index.php/jhnpeps/article/view/4535. http://dx.doi.org/10.30681/252610104535. 